\title{
Investigating the output monitoring component of event-based prospective memory performance
}

\author{
RICHARD L. MARSH \\ University of Georgia, Athens, Georgia \\ JASON L. HICKS \\ Louisiana State University, Shreveport, Louisiana \\ and \\ THOMAS W. HANCOCK and KIRK MUNSAYAC \\ University of Georgia, Athens, Georgia
}

\begin{abstract}
The goal of this study was to augment the standard event-based prospective memory paradigm with an output monitoring component. That component involves memory for past actions and, in the context of prospective memory, is largely responsible for repetition and omission errors. The modified paradigm also provides an index of what people believe to be true concerning their past prospective memory performance. More elaborate prospective responses decreased forgetting that an intention had been fulfilled, whereas contextual change increased forgetting. In Experiments 1-3, people often reported that they had fulfilled an intention on a previous occasion when they actually had not, but distinctive responses reduced that error in Experiment 4. Therefore, people's beliefs about their past performance can influence the incidence of repetition and omission errors in event-based prospective memory tasks.
\end{abstract}

People establish a rich variety of intentions that they wish to accomplish at some later point in time. Collectively, the investigation of intention formation and intention completion is labeled the study of prospective memory (for reviews, see the edited volume by Brandimonte, Einstein, \& McDaniel, 1996). Obviously, not every intention that a person establishes will be completed eventually. Some intentions are forgotten, some are cancelled, and some become obsolete by virtue of environmental change over time. Marsh, Hicks, and Landau (1998) investigated people's everyday intentions and the reasons that they provided for neglecting to complete various types of them. One interesting outcome was that the majority of unfulfilled intentions were not overtly forgotten, as is commonly assumed in the prospective memory literature. Rather, the intentions were cancelled, reprioritized as less important than other obligations, or became impossible to fulfill as a consequence of external factors.

Of course, there are additional reasons for why people's intentions go unfulfilled that were not captured in our earlier study. For example, a person may come to believe that an intention has already been fulfilled, when in fact it has not. The ultimate fate of such an intention is that it will

We thank Allison Smith and Rachel Words for their help in collecting the data. Correspondence should be addressed to R. L. Marsh, Department of Psychology, University of Georgia, Athens, GA 306023013 (e-mail: rlmarsh@uga.edu).

-Accepted by previous editorial team likely go uncompleted unless some environmental stimulus (e.g., one's spouse, secretary, colleague, etc.) serves as a reminder that the intended activity was never performed. On the other hand, the converse situation can also occur. One can believe that one has not completed an intention when the activity has already been performed. This sort of memory failure can lead to repetitions in which a task is carried out (or attempted) more than once. Both sorts of prospective memory errors may have important behavioral consequences, such as over- or undermedicating oneself (e.g., Park, Hertzog, Kidder, Morrell, \& Mayhorn, 1997).

We are not the first, however, to make these observations. Einstein, McDaniel, Smith, and Shaw (1998) developed a paradigm of prospective memory that can be used to study such omission and repetition errors. In their task, participants were engaged in 11 brief activities each lasting $3 \mathrm{~min}$. During each activity, participants had to remember to make a prospective response some time after the first $30 \mathrm{sec}$ had elapsed. Participants were queried directly after each activity as to whether they had successfully remembered to perform the prospective task during the most recent interval. The results indicated that both younger and older adults can become confused about whether an intention has been fulfilled, which can, in turn, lead either to repetition or to omission errors. Einstein et al. (1998) correctly identified the locus of such errors as being the consequence of failing to engage in accurate output monitoring processes (e.g., Koriat, Ben-Zur, \& Sheffer, 1988). Output monitoring is loosely defined as one's memory for one's past actions. In its original context, output 
monitoring was defined as one's memory for what one free recalled or recognized on an earlier memory test (e.g., Gardiner \& Klee, 1976; Klee \& Gardiner, 1976).

In the context of prospective memory, output monitoring failures will occur when one forgets that one has already fulfilled an intention, thereby leading to repetition errors. By contrast, output monitoring failures in which one erroneously believes that one has already performed a task will lead to omission errors. ${ }^{1}$ Einstein et al.'s (1998) paradigm is important because it alerts researchers to the fact that prospective memory can fail in ways other than when research participants fail to make a response as instructed (i.e., overt forgetting or not noticing an event related to an intention). At the same time, Einstein et al.'s (1998) paradigm also begins to broach the important point that successful prospective memory in some circumstances can depend on an important internal state-namely, what one believes to be true of one's past performance (i.e., output monitoring).

In the present study, we modified the existing event-based prospective memory paradigm so that it would require output monitoring in addition to prospective memory. In an eventbased prospective task, people are busily engaged in an ongoing task (e.g., Einstein, Holland, McDaniel, \& Guynn, 1992; Einstein \& McDaniel, 1990). In this context, people were asked to press a particular key when they encountered an exemplar of a certain class of items (e.g., animals). Remembering to press the key is the prospective memory task (e.g., Einstein, McDaniel, Richardson, Guynn, \& Cunfer, 1995; Marsh \& Hicks, 1998; McDaniel \& Einstein, 1993). Presumably, the paradigm is representative of a class of intentions that people establish when they allow cues in the environment to remind them to accomplish various tasks (e.g., letting a grocery store remind one that it should be entered in order to replenish milk).

In the experiments conducted here, we changed the prospective memory task. Event-based cues were repeated in the ongoing task (making pleasantness ratings on a series of words), and our new instructions stated that when an animal word was encountered, the participants should first press a particular key (the prospective response) and then make their pleasantness rating. However, if they remembered having pressed a key to that word earlier, they should press a second, different key to acknowledge that they had already responded earlier to that particular event. Hereafter, we will refer to these keys as the first key and the repeat key. The first four prospective cues were each repeated once, intermingled with four brand new prospective cues for a total of 12 prospective memory trials in a long sequence of 300 words.

The understanding of whether participants can remember their having responded to the earlier repeated targets is theoretically informative insofar as output monitoring is likely to be an important component of the cognitive processing that subserves prospective memory. Currently, very little is known about this retrospective memory component of prospective memory, and our goal was to begin to remedy this. Upon encountering a repeated target, sev- eral outcomes could occur. First, if the target was missed earlier, participants could claim that they had missed it by pressing the first key, or they could incorrectly report that they had responded earlier by pressing the repeat key. Of course, for the targets that were missed the first time, a first keypress to the repetition does not definitively distinguish whether the participants have any memory for the original occurrence or whether they remember their earlier nonresponse; it is simply correct responding. (However, this issue will be addressed in Experiment 2.) Second, if the target had been responded to earlier with a first keypress, participants could acknowledge remembering their response by pressing the repeat key, or they could forget that they had responded and press the first key for a second time in a row to that target. Under any of these scenarios, participants may also omit a response altogether because they failed to notice that the target animal word was related to the intention to respond.

The present design potentially extends the event-based task one step closer to real-world situations in which people must make decisions about whether to engage in an action based on their retrospective memory for having already completed (or not) the task. One may wonder how the present design improves on Einstein et al.'s (1998) directly querying participants about whether they had completed a task. The direct query approach might affect performance by raising the importance of the task (e.g., Kliegel, Martin, McDaniel, \& Einstein, 2001). After all, Einstein et al.'s (1998) younger adults were very close to being at ceiling performance. Asking participants to press a different key when they successfully completed the task earlier does not serve as a constant and pervasive reminder throughout the task. Moreover, the present design begins to investigate how reality monitoring can affect prospective memory. In reality monitoring, people must be able to distinguish between events that have actually happened versus events that they only imagined to have happened (e.g., Johnson, Raye, Foley, \& Foley, 1981). Admittedly, the present paradigm might not apply to all event-based prospective memory tasks, but as we discuss later, the present experiments are certainly applicable to habitual prospective memory tasks that must be repeated (e.g., feeding a pet, taking medication, etc.).

\section{EXPERIMENT 1}

The purpose of Experiment 1 was to obtain some objective measure of how often people remember having responded earlier and how often they forget having done so. In a control condition, which we have labeled the simple condition in all of the experiments, the participants were instructed to press the first key when an animal word was encountered during the cover task. However, if they remembered having responded earlier to that particular animal with a first keypress, they were asked to press the repeat key. Although the data from such a condition would be of interest in its own right, we added a second condition that was intended to make the prospective response 
more memorable. We had the participants say the animal word aloud to the experimenter with the prediction that when they encountered the repetition, this might improve their memory for their original performance (i.e., the first keypress). This condition has been labeled the elaborated condition. Finally, in order to determine whether the lag between the first occurrence and its repetition might affect performance, two repeated targets occurred at a lag of 100 trials and two occurred at a lag of 200 trials.

\section{Method}

Participants. Seventy University of Georgia undergraduates volunteered in exchange for partial credit toward a course research requirement. Assignment to the simple and elaborated conditions was determined by arrival at the laboratory, and an equal number of people $(n=35)$ was tested in each condition. Each participant was tested individually in sessions that lasted approximately $40 \mathrm{~min}$.

Materials. Two hundred eighty-eight concrete nouns were selected from the Kučera and Francis (1967) normative compendium. These were combined with 12 words naming animals (e.g., cow). For any given participant, the software randomly assigned the 288 nonanimal words to the distractor trials. For the prospective memory trials on which an animal word appeared, 8 unique animal words out of the pool of 12 were randomly chosen anew for each participant tested. Prospective memory trials appeared at even intervals of 25 trials, and they occurred on trials $22,47,72,97,122,147$, and so forth, through trial 297 out of a 300 trial test sequence. The assignment of unique and repeated animal words can be denoted by the simple short hand, 1, 2, 3, 4, 1, 2, 5, 6, 7, 8, 3, 4. Thus, each unique number corresponds to a unique animal word, with the first four prospective targets repeated later in the test sequence. Items 1 and 2 were repeated at a lag of 100 trials, and Items 3 and 4 were repeated at a lag of 200 trials.

Procedure. The participants read instructions from the computer monitor. These instructions stated that we were interested in how people judge the pleasantness of certain concepts. To these ends, they would be rating a long list of words for how pleasant the concept was using a 5-point Likert scale. The participants were asked to use the number keys across the top left-hand side of the keyboard so that the rating keypresses would be spatially distinct from the keys used to make a prospective response. The instructions went on to say that we were also interested in their ability to remember to perform an action later. The participants were instructed to press the "/" key when they encountered an animal word. They were further instructed that if they could remember having pressed the " $/$ " key earlier to a particular animal word, they should press the " $=$ " key to indicate that they remembered having pressed the "/" key before to this particular animal. These keys represent the first and repeat keys, respectively, and they are also spatially distinct on the keyboard. The instructions were carefully and unambiguously worded so that the repeat key should only be pressed when the participant could remember having pressed the first key before to that particular target.

The experimenter briefly recapitulated the instructions for both the pleasantness ratings and the prospective memory task to ascertain that the participant had a complete understanding of both tasks. In the elaborated condition, the experimenter also stated verbally that when an animal word was encountered, in addition to pressing a key, the participant should say the word out loud to the experimenter. The experimenter then went on to explain verbally, in both conditions, that there was one short task that needed to be accomplished before the pleasantness rating task. This task was merely a distractor task placed in between the prospective instruction and the onset of the rating task so that the prospective task did not become a vigilance task. The task was a 2 and 7 crossing-out task that required the participants to cross out the numerals 2 and 7 from long lists of random numbers. The distractor task lasted 3 min (timed by stop watch), at which point the pleasantness task began, without reference to any of the instructions. The software kept track of all keypresses. Although the participants could press a first key or repeat key on any trial, they very rarely did so by responding a trial or two late subsequent to the presentation of an animal word (less than $0.5 \%$ ). Therefore, a prospective response was counted only when it preceded the pleasantness rating, which was in accord with our instructions.

\section{Results and Discussion}

Unless otherwise specified with a probability value, statistical significance in each analysis reported did not exceed chance by $5 \%$. We first discuss performance on the nonrepeated targets which includes the first occurrence of Items 1-4 and Items 5-8 which were never repeated (numerals are given in reference to the shorthand notation from the Method section). Repetition performance on Items 1-4 will be handled separately and subsequently. Overall performance was calculated without regard to the key used to make a response. However, it was extremely rare for a participant to press the repeat key to the first occurrence of a target (less than $2 \%$ of the time across all experiments reported herein). The results do not change no matter how these rare events are treated. Average performance as the proportion of targets responded to in the simple condition was .54 $(S E M=.04)$, and in the elaborated it was .73 $(S E M=.03)[t(68)=3.86]$. Thus, making a more elaborated response benefited prospective memory either because the intention was more deeply encoded at the time it was formed or perhaps because oral responses served as better reminders to respond when they were executed over trials. $^{2}$

Before we present the data for repeated targets, we wish to address first a data analytic issue concerning them. There was a small, but nonetheless significant, proportion of participants who either failed to respond to any of the first four prospective memory trials (i.e., targets that were later repeated) or they did not miss any of the first four targets. Because performance is conditionalized on whether the participants had originally missed or had made a response to the first occurrence of repeated targets, this raises the dilemma of whether or not these values should be replaced with a zero. Replacing these values with zero will artificially deflate mean proportions and will also result in proportions that do not quite add up to unity across all possible outcomes. The alternative (and the method we have chosen) is to exclude the participants who did not contribute to one or the other of these conditionalized measures. Therefore, the sample sizes will be reduced for these conditionalized scores and will fluctuate slightly. Fortunately, the overall conclusions do not change with either method of analysis. For this reason, the degrees of freedom in the statistical analyses will vary, depending on the number of participants contributing. Across all conditions in all experiments, this loss of participants was between 2 and 8 people per condition.

The upper half of Table 1 contains the data for the repeated targets when the participants successfully made a 
Table 1

Proportion of Responses Issued to the Repeated Targets Conditionalized on Performance to the First Occurrence in Experiment 1

\begin{tabular}{llllll}
\hline & \multicolumn{4}{c}{ Assigned Condition } \\
\cline { 2 - 3 } \multicolumn{1}{c}{ Responses } & \multicolumn{2}{c}{ Simple } & & \multicolumn{2}{c}{ Elaborated } \\
\cline { 2 - 3 } \cline { 5 - 6 } \cline { 5 - 6 } & Prop. & $S E$ & & Prop. & $S E$ \\
\hline Made first response & & & & \\
Correct: Pressed repeat & .70 & .07 & & .88 & .04 \\
Incorrect: Pressed first & .18 & .05 & & .06 & .04 \\
Omit: No keypress & .12 & .05 & & .06 & .02 \\
Omitted first response & & & & \\
Correct: Pressed first & .35 & .08 & & .16 & .07 \\
Incorrect: Pressed repeat & .49 & .08 & & .59 & .09 \\
Omit: No keypress & .16 & .05 & & .25 & .07 \\
\hline
\end{tabular}

prospective response to the first occurrence of the items. The proportions sum to unity. As can be seen, the participants in the elaborated condition correctly identified these targets as repetitions by pressing the repeat key more often than in the simple condition. Conversely, the participants in the simple condition more often omitted a response or incorrectly labeled the item as a first occurrence despite their having actually responded to it earlier. In a $2 \times 2$ analysis of just the correct claims and the incorrect claims across both conditions, the interaction term was significant $[F(1,68)=5.21]$. Analyses of the simple effects demonstrated that more of the participants incorrectly believed that a repeated item was the first encounter of the target in the simple condition as compared with the elaborated condition (.18 vs. .06) $[t(68)=1.93$, $p=.058]$. Analogously, more of the participants correctly believed that a repetition had been identified earlier in the elaborated condition, as compared with the simple condition (.88 vs. .70) $[t(68)=2.25]$. We had predicted that by making the prospective response more elaborate, the participants would be provided with richer memory traces of their past performance. This outcome occurred and suggests that the output monitoring component of prospective memory benefits from completing more elaborated intentions.

The data in the lower half of Table 1 correspond to the participants' beliefs about the repeated targets when they failed to make a prospective response the first time. Because the participants missed approximately $50 \%$ of these earlier responses, there are approximately equal amounts of data in the upper and lower halves of Table 2 for the simple condition. There were fewer data in the elaborated condition for omissions of first responses, and thus interpretations must be drawn somewhat cautiously in that condition. Nevertheless, the notable aspect to these data is that the participants were incorrectly claiming at very high rates that they remembered having pressed the first key to these repeated targets (by pressing the repetition key). In fact, they had never pressed the first key originally. In an analysis of variance (ANOVA) similar to that conducted previously, only the effect of which key was pressed (first vs. repeat) emerged as significant $[F(1,56)=$
7.51]. Therefore, in both conditions, the participants' misbeliefs that they had responded before exceeded their correct claims that they had not responded to the original occurrence.

As the reader will recall, two of the repetitions occurred at a lag of 100 trials, and two occurred at a lag of 200 trials. Analyses were conducted to determine whether the effects differed in any manner on the basis of the length of the lag between repetitions. Although this might have been a promising manipulation, lag was never a significant variable in this experiment or in the subsequent ones. With only two repetitions at each lag, we may not have had enough power to detect differences. However, these analyses pooled over the between-subjects manipulation for a total of 70 participants, and there was no indication that lag mattered. Lag will not be considered any further.

In general, the results from this experiment indicate that when people make an event-based response, they generally remember having done so, although there is some amount of forgetting (18\%) that appears to be slightly remedied by the completion of more elaborate intentions (upper half of Table 1). By contrast, when the participants missed a response, they often believed that they had responded earlier when actually they had not (lower half of Table 1). Thus the participants tended to behave as if they had performed an action both when it was performed earlier and when it was not performed. The data suggest that people are remembering an item to have occurred, but not that the appropriate action was omitted originally. Because the action was not taken earlier, they cannot be remembering having pressed the first key. Rather, this misbelief that they had responded earlier could be the result of an inference along the lines of "If I remember having seen this item, I can assume that I had processed it appropriately the first time." In the early stages of this research, we had considered the possibility that the participants might have misunderstood our instructions and believed that the repeat key meant that an item had merely been encountered earlier, regardless of their prospective memory performance. This was not the instruction delivered, nor was it the belief of the participants that we debriefed at the end of the experiment. The vast major-

Table 2

Proportion of Responses Issued to the Repeated Targets Conditionalized on Performance to the First Occurrence in Experiment 2

\begin{tabular}{ccc}
\hline & \multicolumn{2}{c}{ Simple Condition } \\
\cline { 2 - 3 } Responses & Prop. & $S E$ \\
\hline Made first response & & \\
Correct: Pressed repeat & .67 & .09 \\
Incorrect: Pressed first & .28 & .09 \\
Omit: No keypress & .03 & .02 \\
Omitted first response & & .10 \\
Correct: Pressed first & .31 & .11 \\
Incorrect: Pressed repeat & .46 & .09 \\
Omit: No keypress & .20 & \\
\hline
\end{tabular}


ity of the participants (over 96\%) correctly stated that the repeat key was to be pressed if they had a memory for having pressed the first key earlier to that item. In addition, all the participants could correctly identify which keys were to be pressed when they were asked to identify them during debriefing. The participants might have been confused about their earlier actions, but they were not confused about the requirements of the task that they had been asked to perform.

\section{EXPERIMENT 2}

The purpose of this next experiment was to investigate an ambiguity about participants' memory for previously encountered targets (i.e., repeated items). When a target was responded to earlier, a participant's issuing a repeat response decidedly indicates memory for both the event and the earlier prospective response. The same is true when the target is missed and a repeat response is issued. In the latter case, the participant correctly remembers an item to have occurred but incorrectly believes that a prospective response had been given earlier. Thus, there is no ambiguity about the interpretation of what a participantbelieves when the repeat key is pressed. The same is not true when the participant presses the first key to repeated targets. When a target receives a successful response on a previous occasion, a participant's issuing a first key response could indicate either (1) that the participant believes no response to have been issued before (forgetting the prospective response) but remembers the item to have occurred, or (2) that the participant has forgotten both the item and having made the prospective response. Similarly, when a target was missed on a previous occasion, a participant's pressing the first key does not disambiguate whether the participant remembers the item to have occurred (and realizes no response had been issued earlier) or has forgotten that the item had been encountered at all. It is important to understand what participants believe about these first responses, because it is an important aspect of the output monitoring component of prospective memory and of the paradigm we have developed to study it. ${ }^{3}$

To investigate these first responses, the simple condition of Experiment 1 was repeated in this experiment, with one additional feature. Any time the first key was pressed, the software controlling the experiment prompted the participant to indicate (yes or no) whether the word on the current trial had occurred previously in the experiment. Although this query had the potential to affect both prospective memory and output monitoring performance, this methodology provides some insight into whether (1) the participants were forgetting that an item had occurred, or (2) are claiming that it had occurred, but they believe they did not respond to the intention successfully before. Because this query is a form of an elaborated response, we anticipated that performance could have been better than in the simple condition of Experiment 1.

\section{Method}

Participants. Twenty-eight volunteers from the same pool as in Experiment 1 were awarded research credit in exchange for participating. Each participant was tested individually in sessions that lasted approximately $45 \mathrm{~min}$.

Procedure. The procedure was identical to that in the simple condition of Experiment 1, with one exception. Any time a participant pressed the first key, a query appeared two lines beneath the word asking, "Did this word occur before? $\mathrm{Y}=$ yes, $\mathrm{N}=$ no." The participant had to respond either yes or no before proceeding to enter a pleasantness rating and moving on to the next trial. At the end of the experimenter's recapitulation of the instructions, the participants were warned that they might occasionally receive such a query. As before, the distractor task followed the instructions and occurred before the commencement of the ongoing task.

\section{Results and Discussion}

The proportion of trials on which a prospective memory response was issued to the nonrepeated targets was .73 $(S E M=.04)$. This average is higher than that in the simple condition of Experiment 1 (.54) and is directly comparable with the elaborated condition of that experiment. We had anticipated that by making an additional judgment whenever the first key was pressed, prospective performance would slightly increase by highlighting the prospective task in the context of the ongoing task. For these nonrepeated targets, the participants responded $98 \%$ of the time that they had not encountered the target previously. This outcome indicates that the participants were not becoming confused concerning individual items within the animal category and that they could easily differentiate the occurrences of individual targets from one another.

Performance on the repeated targets is summarized in Table 2. Replicating Experiment 1, when the participants made a prospective response previously (upper half of Table 2), they largely remembered having done so, but they did sometimes forget, as indicated by the proportion of first keypresses. Obviously, correct issuance of the repeat key was greater than the forgetting, indicated by incorrect first keypresses $[t(25)=2.23]$. The purpose of this experiment was to determine whether the participants had forgotten that the item had occurred or whether they simply had forgotten their previously correct prospective response. Of the $28 \%$ of the cases in which a first key was pressed, all $28 \%$ of the targets on these trials were claimed to have been experienced earlier. In other words, the data indicate that in $100 \%$ of the cases, the participants remembered the item but had forgotten their previous successful prospective response.

Performance on the targets for which the prospective response was missed on its first occurrence is summarized in the lower half of Table 2 . These data are directly comparable with those of the simple condition of Experiment 1, probably because no special response had been made earlier (i.e., they were missed). As in the first experiment, the erroneous beliefs of the participants' having performed the prospective response numerically exceeded cases in which the participants correctly pressed the first key. However, this effect was not statistically significant, with fewer par- 
ticipants contributing to the analysis $[t(24)<1.0$, n.s.]. In response to the query about whether they had seen these items previously, the vast majority $(84 \%)$ responded in the affirmative. Therefore, even when targets did not receive a prospective response previously, the participants remembered that they had encountered the item before. Only an average of $16 \%$ of the time did they indicate that the item had not been seen before, and these more infrequent claims obviously reflect item forgetting.

In summary, the results from this experiment indicate that the participants pressed the first key because they believed that they have not responded earlier in accord with their intention to do so. Very few of the first responses indicated that the participants had forgotten the occurrence of the item itself. This never happened when they successfully responded previously, and this occurred only in a small minority of cases when they had entirely missed making a response on the first occurrence. As a consequence, we can have some confidence in this paradigm that first keypresses generally indicate the forgetting of the prospective response (when it was made) or correct identification that one had forgotten to make the response as intended (when it was omitted). Having clarified the interpretation of first keypresses, we now report another manipulation designed to affect the output monitoring component of event-based prospective memory.

\section{EXPERIMENT 3}

In this next experiment, we tested another factor (besides elaboration) that might be expected to change the output monitoring component of event-based prospective memory. McDaniel, Robinson-Riegler, and Einstein (1998) demonstrated that when the context surrounding an intention is changed from the time the intention is formed to the time when it is appropriate to execute the task, prospective memory performance declined, as compared with when the context remained the same. If this result generalizes to the paradigm developed here, changing the context from the time an item is first encountered to the time it is repeated could weaken one's memory for the item and any actions that were (or were not) associated with it. Of course, such a prediction is consistent with the transfer appropriate processing literature as well (e.g., Basden, Bonilla-Meeks, \& Basden, 1994; Challis \& Sidhu, 1993). The important point is that standard variables affecting retrospective memory should change output monitoring; and the issue under scrutiny here is how such manipulations of retrospective memory for actions might affect prospective memory.

Therefore, half of the participants assigned to the simple, control condition made pleasantness ratings on all 300 items, and the other half made imageability ratings (i.e., they rated how concrete an image could be formed). In the experimental condition, the participants were given a break after 150 trials, and they changed the processing task they were doing from pleasantness ratings to image- ability ratings at this break (or vice versa, depending on counterbalancing). These two tasks were chosen in pilot testing in order to yield equivalent overall prospective memory performance. We predicted that if the context changed from the first encounter to the second (repeated) encounter of a particular target, one might be more likely to forget that one had responded earlier (i.e., forget a prospective response). Similarly, such an effect might also reduce the false claims of having responded earlier because the context change might cause one to forget that an item had occurred earlier.

\section{Method}

Participants. Seventy volunteers who had not participated previously were recruited. They were awarded course credit toward fulfilling a research requirement. Thirty-five were tested under essentially the same simple, control condition as that in Experiment 1, and the remaining participants were tested in the experimental condition, which we have labeled the context condition to denote that target repetitions were encountered under a different ongoing task requiring different cognitive processing.

Procedure. In the control condition, approximately equal numbers of participants were tested using either the pleasantness rating cover task or the imageability cover task. In both cases, the participants made their judgments on a 5-point Likert scale. These participants were given a break after 150 trials, corresponding to the length of time to administer instructions in the context condition. In the context condition, the participants started by making pleasantness ratings on the first 150 trials and then were switched with verbal instructions to judge the remaining 150 items for imageability, or the order of the tasks was reversed. In order to preserve four repeated targets in the second half of the sequence, the sequence of prospective trials in both conditions was $1,2,3,4,5,6,1,2,7,8$, 3,4 , according to the shorthand notation used in the Method section of Experiment 1. In all other respects, the experiment was identical to the simple condition of Experiment 1.

\section{Results and Discussion}

The data from this experiment were first analyzed to determine whether the two ongoing tasks that were used affected overall prospective memory performance. In none of those analyses did the two tasks differ, nor did that variable interact in any significant or meaningful way with other variables of interest. Therefore, this factor is not considered any further. Average performance on the nonrepeated targets was .58 $(S E M=.05)$ in the simple condition and $.62(S E M=.04)$ in the context condition $[t(68)<1.0$, n.s. $]$. This overall performance translates into approximately $60 \%$ of the data's being analyzed in the conditionalized analyses for trials on which the participants remembered to press the first key to first occurrences and the remaining $40 \%$ for conditionalized performance when they forgot to make that response.

The data for the repeated prospective targets are set forth in Table 3. For the trials on which the participants remembered to make an initial prospective response (upper half of Table 3), the results generally replicated those in Experiment 1 , insofar as the participants generally remembered having made that earlier response by correctly pressing the repeat key. Less frequently they forgot that they had made 
Table 3

Proportion of Responses Issued to the Repeated Targets Conditionalized on Performance to the First Occurrence in Experiment 3

\begin{tabular}{llllll}
\hline & \multicolumn{4}{c}{ Assigned Condition } \\
\cline { 2 - 3 } \cline { 5 - 6 } \multicolumn{1}{c}{ Responses } & \multicolumn{2}{c}{ Simple } & & \multicolumn{2}{c}{ Context } \\
\cline { 2 - 3 } \cline { 5 - 6 } & Prop. & $S E$ & & Prop. & $S E$ \\
\hline Made first response & & & & \\
$\quad$ Correct: Pressed repeat & .78 & .06 & .59 & .07 \\
Incorrect: Pressed first & .15 & .06 & .30 & .06 \\
Omit: No keypress & .07 & .04 & .10 & .03 \\
Omitted first response & & & & .03 \\
Correct: Pressed first & .39 & .07 & & .42 & .08 \\
Incorrect: Pressed repeat & .54 & .08 & .45 & .09 \\
Omit: No keypress & .06 & .03 & .13 & .05 \\
\hline
\end{tabular}

the prospective response and pressed the first key. The 2 (correct vs. incorrect keypress) $\times 2$ (simple vs. context condition)ANOVA supported this observation $[F(1,60)=$ $31.1]$. There was no main effect of condition, but the interaction was significant $[F(1,60)=4.35]$. The obvious interpretation of these data in conjunction with the results of Experiment 2 is that the change in context increased the participants' forgetting of having made the prospective response, as opposed to their forgetting that the item had been encountered previously. This thesis accords quite well with the idea that contextualchange usually does not affect recognition-based processes but does affect recall-based processes (e.g., Russo, Ward, Geurts, \& Scheres, 1999). Therefore, the participants most probably recognized that the target had occurred earlier, but could not recall whether they had responded appropriately. On the whole, the data are consistent with the notion that reinstating a particular context will help a rememberer to decide whether a prospective event had been responded to previously.

The data for the prospective trials that had been missed on their first occurrence are summarized in the lower half of Table 3. All three $F$ values in the $2 \times 2$ ANOVA were less than 1 . Therefore, the general conclusion is that the two conditions did not significantly differ in the participants' correct claims that this was the first time that they had responded, nor in their incorrect claims that they had responded before. The reasonable interpretation of these outcomes is that the participants believed that they had responded earlier (when they had not) just as frequently as they correctly identified that they had not responded earlier. Therefore, the consistent aspect to the data from all three experiments is that the participants may generally believe that their own prospective memory performance was better than it really was.

By way of summary, the contextual change increased the participants' forgetting of having made a successful response before. That result suggests that contextual change could increase the probability of a repetition error after successful intention completion. Just as contextual reinstatement helps some forms of retrospective memory such as free recall, so too it helps the output monitoring component of remembering whether one has performed an in- tended action. By contrast, a contextual change does not seem to affect one's memory for having missed an earlier target. In retrospect, these outcomes make some intuitive sense, insofar as context should be more easily associated with an event that had occurred in the past (making a response) as opposed to the absence of an event (failing to make a response). Nevertheless, when the original target was missed, the participants often believed that they had responded to the first occurrence even though they had not.

\section{EXPERIMENT 4}

One potential shortcoming of the paradigm developed here is that the participants are always initiating the same action with the same key in order to make a first response to each target. Although Experiment 2 demonstrated quite clearly that people do remember the individual targets fairly well, they might be confusing pressing the first key to one particular animal versus another. By this analysis, the target items themselves are fairly distinct, but there could exist some confusion in a participant's memory about the original prospective response. ${ }^{4}$

To investigate this issue concerning the erroneous beliefs that missed targets were responded to appropriately, we changed the nature of the first keypress in this next experiment. Rather than having the participants press a single key to indicate successful prospective memory, the new instruction was to press the key corresponding to the beginning letter of the animal target word (e.g., $d$ for $d o g, l$ for lion, etc.). Potentially, this manipulation should make the original prospective response more distinctive among the various targets that are successfully identified. By the same token, if one missed a target originally and cannot remember clearly whether a response had been made, determining whether a unique letter had been pressed might aid in deciding that pressing a first key (i.e., the first letter of the target) is the appropriate response rather than pressing the repeat key. In this case, the absence of a memory for a more distinctive action could aid the output monitoring component of this task and result in fewer erroneous beliefs of having completed an action that had never been performed (cf. Strack \& Bless, 1994).

\section{Method}

Participants. Seventy volunteers from the same pool as in the previous experiments were recruited and awarded course research credit in exchange for participating. Each participant was tested individually.

Procedure. As in the previous experiments, a simple, control condition was tested for comparison with what will be labeled the distinctive condition. In the distinctive condition, the participants were to press the key that corresponded to the beginning letter of the target whenever they encountered an animal word during the pleasantness rating task. Several changes were made to the pool of 12 animal words to ensure that all of them began with a unique letter of the alphabet. Recall that a given participant experienced only a randomly determined 8 of the pool of 12 targets. The repeat key was the same as it had been in the previous experiments. Thus, the participants in the simple condition pressed one of two prospective keys, whereas those assigned to the distinctive condition pressed 
Table 4

Proportion of Responses Issued to the Repeated Targets Conditionalized on Performance to the First Occurrence in Experiment 4

\begin{tabular}{llllll}
\hline & \multicolumn{4}{c}{ Assigned Condition } \\
\cline { 2 - 3 } \multicolumn{1}{c}{ Responses } & \multicolumn{2}{c}{ Simple } & & Distinctive \\
\cline { 2 - 3 } \cline { 5 - 6 } & Prop. & $S E$ & & Prop. & $S E$ \\
\hline Made first response & & & & \\
$\quad$ Correct: Pressed repeat & .71 & .06 & .76 & .06 \\
Incorrect: Pressed first & .17 & .05 & .19 & .06 \\
Omit: No keypress & .12 & .05 & .04 & .04 \\
Omitted first response & & & & .06 & .09 \\
Correct: Pressed first & .43 & .09 & & .66 & .09 \\
Incorrect: Pressed repeat & .37 & .08 & .24 & .08 \\
Omit: No keypress & .20 & .07 & .10 & .05 \\
\hline
\end{tabular}

one of many different keys on their first encounter with an animal word and the same repeat key if they remembered having responded earlier. In all other respects the procedural details were identical.

\section{Results and Discussion}

The average proportion of trials on which a prospective response was made to the nonrepeated targets was .67 $(S E M=.04)$ in the simple condition and $.72(S E M=.04)$ in the distinctive condition. Obviously, the pressing of a single key versus the pressing of many different ones did not greatly change the overall rate at which targets were identified $[t(68)<1.0$, n.s. $]$. Although the slightly more complex intention in the distinctive condition could have increased overall prospective memory, it did not. The data for the repeated targets is reported in Table 4. The top half of that table summarizes performance for the second encounter of targets that originally received a successful response. Performance in the simple condition nicely matched the results from the previous experiments. The participants generally remembered their correct previous response and much less frequently forgot that they had responded. Contrary to our predictions, the pressing of different keys in the distinctive condition did not change performance very much at all. The statistical analyses confirmed these observations. More responses reflected successful recall of having made the previous response (i.e., a repeat keypress), rather than forgetting having made the response (i.e., a first keypress) $[F(1,63)=$ 57.22]. There was no main effect of assigned condition, nor an interaction (both $F_{\mathrm{S}}<1.0$, n.s.). Thus, distinctive keypresses on the first encounter did not aid the participants in remembering that a previous action had been completed.

The results for the targets that had been missed originally are reported in the lower half of Table 4 . Recall that in the previous experiments, the participants either displayed equal levels of correct responses (first key) and errors of erroneously believing they had responded (repeat key), or they displayed significantly more erroneous beliefs than correct ones. In this experiment, the reverse was true. Correct identifications that this was the first response statistically exceeded claims of erroneous pre- vious performance $[F(1,55)=9.72]$, and that outcome is largely a consequence of the much reduced error rate in the distinctive condition. Although the interaction term in the $2 \times 2$ ANOVA failed to reach conventional significance $[F(1,55)=2.56, p>.11]$, the simple effect of type of key pressed was not significant in the control condition $[t(57)<1.0]$ but was in the distinctive condition $[t(57)=2.23]$. Therefore, the participants' making distinctive responses to the targets originally reduced erroneous beliefs about their having completed an intention when they had not.

The results of this experiment suggest that the retrospective memory associated with the output monitoring component for previously missed opportunities can be improved. The lack of a memory for a somewhat unique action aided the participants in determining that they had not responded previously. In the previous experiments, memory for completed actions was more tractable to experimental manipulations, but this experiment demonstrated that the lack of diagnostic evidence in memory from one's having missed an earlier opportunity to respond can increase later correct responding in this prospective memory task (cf. Strack \& Bless, 1994). Because the memory associated with output monitoring determines people's beliefs about their past performance, we have found several new variables that might be likely to influence real-world performance. We turn now to consider what these experiments demonstrate more generally about event-based prospective memory.

\section{GENERAL DISCUSSION}

The results of these experiments suggest that prospective memory failures can occur because of one's own beliefs concerning one's past performance. In other words, retrospective memory should play a more critical role in theories of prospective memory beyond its current portrayal as being the ability to remember the content of an intention. This message is a relatively novel one that comes directly from the hypothesis that successful prospective memory involves not only one's recognition that environmental cues are relevant to an intention stored in memory, but also one's probing memory for retrospective details of output monitoring and one's past performance. These experiments also argue that omission errors can occur for reasons other than memory or stimulus identification errors-namely, one might simply believe that an intention has already been fulfilled. Similarly, repetition errors can occur-not because a previous encounter with a target has been forgotten-but because the successful prospective response has been forgotten in spite of one's remembering the original encounter. These are critical functions of retrospective memory whose presence and influence in theories of prospective memory have hitherto largely been unexplored.

Our new paradigm highlights these important aspects of retrospective memory as being integral to the output 
monitoring component of prospective memory. Nevertheless, we readily admit that the frequency of omission and repetition errors observed in this paradigm might overstate their prevalence when everyday intentions are established outside the laboratory. However, both sorts of errors do occur (e.g., Park et al., 1997), and our understanding the various mechanisms by which they arise is an important component of apprehending event-based prospective memory. The occurrence of these errors with certain intentions might have very serious consequences, such as overmedicating one's self or leaving a hazardous appliance (e.g., a stove or a heater) turned on for an extended period of time. Fortunately, the environment can leave clues so that if a repetition is attempted, the error is usually quickly detected (e.g., one's attempting to grab paid bills on the way out of the house that had been mailed as a part of yesterday's going-to-work activities).

Our thesis throughout has been that intentions are acted on or behavior is withheld as a function of the output monitoring component of prospective memory. As it relates to omission errors, many failures of prospective memory might not be failures at all, but rather might be conscious decisions to withhold behavior on the basis of the belief that an intention has already been carried out. The environment can be less friendly in the case of omission errors than it could be for repetition errors. For example, one usually intends to lock one's car after exiting it. If one erroneously believes that this intention has been fulfilled, the car will go unlocked. The environment might not offer up convenient cues that this activity was missed. Rather, one must explicitly check to see if one's car or house door has been locked.

Of course, these observations are somewhat premature in regard to the amount of environmental support that exists for reducing prospective memory errors. There are assuredly some intentions and their associated activities that are more prone to both omission and repetition errors than are other sorts of intentions for which both sorts of errors are less likely to occur. For example, repetitive or habitual sorts of tasks are probably the ones in which an erroneous belief of one's having fulfilled an intention leads to omission errors (Einstein et al., 1998). The unique trouble of tracking habitual intentions is that they are prone to a severe form of proactive interference that causes confusion from past performance. Unfortunately, most of one's memory for habitual tasks is a schematic memory for successful past performances (e.g., picking up dry cleaning at the laundry). If one queries oneself halfway down the driveway about whether the door to the house was just locked, one may be very prone to substitute the schematic memory of what was accomplished on so many prior occasions with a "memory" for having done it on this particular occasion. The same may be true of prominently displaying medication in the middle of the kitchen counter as a reminder to oneself to take it. In these cases, only unique circumstances of the current event (e.g., a key stuck in the lock) can be used as diagnostic evidence that the habitual intention had been fulfilled on this particular occasion. These ideas are consistent with the result that distinctive keypresses in Experiment 4 reduced erroneous beliefs about one's having performed an activity in the past.

Our goal is not to paint a bleak picture of event-based performance that has little chance of success in everyday life. On the contrary, event-based performance is likely to be quite successful in the real world. An eventbased paradigm is simply a microcosm that can be used effectively to study in the laboratory the basic phenomena that are likely to occur in everyday life. The rating of words for pleasantness and remembering to press keys on a keyboard are likely to be simpler tasks than those that humans perform on a daily basis. Thus, the memory traces formed in this laboratory paradigm are probably weaker than the richer, more contextualized memories established by normal human existence. Nevertheless, the errors observed here suggest that omissions and repetitions do occur as a consequence of failures in the output monitoring component of prospective memory. The increase in output monitoring with elaborative responding that an intention had been fulfilled earlier resulted in better prospective memory. These results are very reassuring because they demonstrate that with more complex intentions and more complex contexts (as in everyday life), prospective memory is likely to be more accurate.

At the same time, the results of Experiment 3 are somewhat disheartening if they are truly indicative of real-world prospective memory. In that experiment, a contextual change from the time one encountered and successfully fulfilled an intention to the time a target was re-encountered severely depressed memory of the earlier prospective success. McDaniel et al. (1998) found a similar result with contextual changes between the formation of an intention and the occurrence of the cue creating the demand to respond. Identification of the cue as related to an intention to respond was worse if the context had changed from intention formation to the appropriate time to execute the activity. The unfortunate aspect of these results is that most human intentions are delayed intentions, in which the intention to perform a task later is created because it is not appropriate to perform the activity now or because the conditions for execution are not currently met. If these contextual effects generalize beyond event-based paradigms in which they have been primarily tested, they suggest that intentions formed in one context might not be remembered as well in another (different) context.

The major point of this article is that prospective memory can depend on what people believe to be true about their past performance (i.e., output monitoring). Depending on how veridical that retrospective memory is, errors of omission and repetition can occur and change the probability that intentions will be fulfilled. The corollary to this major point is that most decisions concerning whether an intention is to be fulfilled or whether an action is to be withheld probably involve some consideration as to whether the action has already been fulfilled, and if so, when and under what circumstances it was accomplished. These considerations are likely to be especially important 
with habitual prospective memory tasks. We have investigated variables that have resulted in changes to prospective memory performance based on people's recollections (and thus beliefs) concerning their past performance. Therefore, the present experiments suggest that a consideration of output monitoring issues should be explored more systematically and more generally as they relate to theories of prospective memory (see also Dobbs \& Reeves, 1996, who stress this point as well). Until recently, retrospective memory was considered mainly important for one's remembering the content of an intention. By contrast, this series of experiments has demonstrated a much more important role for retrospective memory in prospective memory processes-namely, as it relates to one's memory for one's past prospective memory performance.

\section{REFERENCES}

Basden, B. H., Bonilla-Meeks, J. L., \& Basden, D. R. (1994). Crosslanguage priming in word-fragment completion. Journal of Memory \& Language, 33, 69-82.

Brandimonte, M., Einstein, G. O., \& McDaniel, M. A. (1996). Prospective memory: Theory and applications. Hillsdale, NJ: Erlbaum.

Challis, B. H., \& Sidhu, R. (1993). Dissociative effect of massed repetition on implicit and explicit measures of memory. Journal of Experimental Psychology: Learning, Memory, \& Cognition, 19, 115-127.

Dobbs, A. R., \& Reeves, M. B. (1996). Prospective memory: More than memory. In M. Brandimonte, G. O. Einstein, \& M. A. McDaniel (Eds.), Prospective memory: Theory and applications (pp. 199-221). Hillsdale, NJ: Erlbaum.

Einstein, G. O., Holland, L. J., McDaniel, M. A., \& Guynn, M. J. (1992). Age-related deficits in prospective memory: The influence of task complexity. Psychology \& Aging, 7, 471-478.

Einstein, G. O., \& McDANIEL, M. A. (1990). Normal aging and prospective memory. Journal of Experimental Psychology: Learning, Memory, \& Cognition, 16, 717-726.

Einstein, G. O., McDaniel, M. A., Richardson, S. L., Guynn, M. J., \& Cunfer, A. R. (1995). Aging and prospective memory: Examining the influences of self-initiated retrieval processes. Journal of Experimental Psychology: Learning, Memory, \& Cognition, 21, 996-1007.

Einstein, G. O., McDaniel, M. A., Smith, R. E., \& Shaw, P. (1998). Habitual prospective memory and aging: Remembering intentions and forgetting actions. Psychological Science, 9, 284-289.

GARDINER, J. M., \& KLEE, H. (1976). Memory for remembered events: An assessment of output monitoring in free recall. Journal of Verbal Learning \& Verbal Behavior, 15, 227-233.

Johnson, M. K., Raye, C. L., Foley, H. J., \& Foley, M. A. (1981).
Cognitive operations and decision bias in reality monitoring. American Journal of Psychology, 94, 37-64.

KLeE, H., \& Gardiner, J. M. (1976). Memory for remembered events: Contrasting recall and recognition. Journal of Verbal Learning \& Verbal Behavior, 15, 471-478.

Kliegel, M., Martin, M., McDaniel, M. A., \& Einstein, G. O. (2001). Varying the importance of a prospective memory task: Differential effects across time- and event-based prospective memory. Memory, 9, 1-11.

Koriat, A., Ben-Zur, H., \& Sheffer, D. (1988). Telling the same story twice: Output monitoring and age. Journal of Memory \& Language, 27, 23-39.

KUČERA, H. \& FrAnCIS, W. N. (1967). Computationalanalysis of presentday American English. Providence, RI: Brown University Press.

MARSH, R. L., \& Hicks, J. L. (1998). Event-based prospective memory and executive control of working memory. Journal of Experimental Psychology: Learning, Memory, \& Cognition, 24, 336-349.

Marsh, R. L., Hicks, J. L., \& LANDAU, J. D. (1998). An investigation of everyday prospective memory. Memory \& Cognition, 24, 633-643.

McDaniel, M. A., \& Einstein, G. O. (1993). The importance of cue familiarity and cue distinctiveness in prospective memory. Memory, $\mathbf{1}$, 23-41.

McDaniel, M. A., Robinson-Riegler, B., \& Einstein, G. O. (1998). Prospective remembering: Perceptually driven or conceptually driven processes? Memory \& Cognition, 26, 121-134.

Park, D. C., Hertzog, C., Kidder, D. P., Morrell, R. W., \& Mayhorn, C. B. (1997). Effect of age on event-based and time-based prospective memory. Psychology \& Aging, 12, 314-327.

Russo, R, WArd, G., Geurts, H., \& Scheres, A (1999). When unfamiliarity matters: Changing environmental context between study and test affects recognition memory for unfamiliar stimuli. Journal of Experimental Psychology: Learning, Memory, \& Cognition, 25, 488-499.

Strack, F., \& Bless, H. (1994). Memory for nonoccurences: Metacognitive and presuppositional strategies. Journal of Memory \& Language, 33, 203-217.

\section{NOTES}

1. In everyday life, it would be difficult to distinguish between beliefs of prior commission and simple forgetting of an intention without directly querying a person. The experimental paradigm that we have developed and that is described in detail later has a natural solution to this problem.

2. Across the experiments, nonrepeated performance on Items 1-4 versus Items 5-8 was comparable. Thus, there was no significant practice effect and the average correct performance reported in this paragraph can be considered representative of both sets of targets.

3. We thank Gus Craik for highlighting this important issue to us.

4. We thank both Gus Craik and Gil Einstein for independently making this point to us. 